\title{
ESTIMATES OF DISJOINT SEQUENCES IN BANACH LATTICES AND R.I. FUNCTION SPACES
}

\author{
R. GONZALO AND J.A. JARAMILLO
}

\begin{abstract}
We introduce $U D S_{p}$-property (resp. $U D T_{q}$-property) in Banach lattices as the property that every normalized disjoint sequence has a subsequence with an upper $p$-estimate (resp. lower $q$-estimate). In the case of rearrangement invariant spaces, the relationships with Boyd indices of the space are studied. Some applications of these properties are given to the high order smoothness of Banach lattices, in the sense of the existence of differentiable bump functions.
\end{abstract}

Key words and phrases. Upper and lower estimates, r.i. spaces, smoothness.

\section{INTRODUCTION}

Recall that, for $1<p, q<\infty$, a sequence $\left\{x_{k}\right\}_{k=1}^{\infty}$ in a Banach space $X$ is said to have an upper p-estimate (respectively, a lower q-estimate) with constant $C>0$ if for any $a_{1}, \ldots, a_{n} \in \mathbb{R}$ and $n \in \mathbb{N}$,

$$
\begin{gathered}
\left\|\sum_{k=1}^{n} a_{k} x_{k}\right\| \leq C\left(\sum_{k=1}^{n}\left|a_{k}\right|^{p}\right)^{1 / p} \\
\text { (resp. } \left.\quad C\left(\sum_{k=1}^{n}\left|a_{k}\right|^{q}\right)^{1 / q} \leq\left\|\sum_{k=1}^{n} a_{k} x_{k}\right\|\right) .
\end{gathered}
$$

According to [19, a Banach space $X$ is said to have property $S_{p}$ if every weakly null normalized sequence in $X$ has a subsequence with an upper $p$-estimate. In a similar way, $X$ is said to have property $T_{q}$ if every weakly null normalized sequence has a subsequence with a lower $q$-estimate (see [16]). These properties play an important role in the behavior of polynomial maps on the space, as can be seen in [16, [7] and [11] and they are also related to high order smoothness of the space ( see [16] and [17]).

Here we study a variant of these properties in Banach lattices and, more specifically, in the class of rearrangement invariant spaces. Note that in the simple case 
of spaces $L_{p}(\mu)$, where $1<p<\infty$, since the space contains an isomorphic copy of $\ell_{2}$, it verifies property $S_{r}$ just for $r \leq \min \{p, 2\}$. On the other hand, every disjoint normalized sequence is equivalent to the unit vector basis of $\ell_{p}$. This remark motivates the study of new properties related to the existence of upper or lower estimates for disjoint sequences. For technical reasons we are interested in the existence of such estimates in an uniform way.

For $1<p, q<\infty$ we say that a Banach lattice $X$ satisfies the uniform disjoint $S_{p}$-property, in short $U D S_{p}$-property, (respectively, uniform disjoint $T_{q}$-property, $U D T_{q}$ - property ) if there exists a constant $C>0$ such that every normalized disjoint sequence in $X$ satisfies an upper $p$-estimate (resp. a lower $q$-estimate) with constant $C$. It is then clear that spaces $L_{p}(\mu)$ for $1<p<\infty$ verify both $U D S_{p}$ and $U D T_{p}$ properties.

To simplify the notation we introduce the following indices:

$$
\begin{aligned}
& \ell_{d}(X)=\sup \left\{p>1: \quad X \text { has } U D S_{p}-\text { property }\right\} \\
& u_{d}(X)=\inf \left\{q>1: \quad X \text { has } U D T_{q}-\text { property }\right\}
\end{aligned}
$$

On the other hand, recall that for $1<p, q<\infty$, a Banach lattice $X$ is said to have a lattice upper p-estimate (respectively, a lattice lower q-estimate) if there is a constant $C>0$ such that, for every finite family of disjoint vectors $x_{1}, \ldots x_{n}$ in $X$, we have

$$
\left\|\sum_{k=1}^{n} x_{k}\right\| \leq C\left(\sum_{k=1}^{n}\left\|x_{k}\right\|^{p}\right)^{1 / p}
$$

(respectively,

$$
\left.\left(\sum_{k=1}^{n}\left\|x_{k}\right\|^{q}\right)^{1 / q} \leq C\left\|\sum_{k=1}^{n} x_{k}\right\|\right) .
$$

We also define:

$$
\begin{aligned}
& u p(X)=\sup \{p>1: X \text { has a lattice upper p-estimate }\} \\
& \text { low }(X)=\inf \{q>1: X \text { has a lattice lower q-estimate }\}
\end{aligned}
$$

Of course, if $X$ has a lattice upper $p$-estimate (respectively, a lattice lower $q$-estimate) then $X$ satisfies the $U D S_{p}$-property (respectively, $U D T_{q^{-}}$property). As can be seen with simple examples, the converse is not true. Indeed, for $1<p, q<\infty$ consider the Banach lattice $X_{p, q}=\left(\bigoplus_{n=1}^{\infty} \ell_{q}^{n}\right)_{\ell_{p}}$ with its natural 
ESTIMATES OF DISJOINT SEQUENCES IN BANACH LATTICES AND R.I. FUNCTION SPACES

order. Using a standard sliding hump argument, it can be shown that every normalized disjoint sequence in $X_{p, q}$ has a subsequence equivalent to the unit vector basis of $\ell_{p}$, with uniform constant. Then $X_{p, q}$ has both $U D S_{p}$ and $U D T_{p}$ properties. On the other hand, in the case $q<p, X_{p, q}$ has no lattice upper $r$-estimate for $r>q$, and in the case $q>p, X_{p, q}$ has no lattice lower $r$-estimate for $r<q$. Thus for a Banach lattice $X$ we have that $u p(X) \leq \ell_{d}(X)$ and $u_{d}(X) \leq l o w(X)$, and these inequalities are strict in general.

In Section 2, we will relate these new properties $U D S_{p}$ and $U D T_{q}$ with Boyd indices $p_{X}$ and $q_{X}$ in the context of separable rearrangement invariant spaces $X(I)$, where $I$ is either $\mathbb{N},[0,1]$ or $[0, \infty)$ (in short, r.i. spaces). Recall that Boyd indices play an important role in interpolation problems, that is, the study of r.i. function spaces which are between $L_{p}(I)$ and $L_{q}(I)$, in the sense that every operator which is bounded in these two spaces, is bounded also on $X(I)$. We prove that if $I=\mathbb{N}$ or $I=[0, \infty)$ and $X(I)$ has $U D S_{p}$-property (respectively, $U D T_{q}$-property), then $p \leq p_{X}$ (respectively, $\left.q \geq q_{X}\right)$. This fact is not true in the case of r.i. function spaces on $[0,1]$ as Proposition 5 below will show.

Section 3 is devoted to study the connection between $U D S_{p}$-property and high order smoothness of Banach lattices. The smoothness of a space is here understood as the existence of bump functions, that is, real valued functions with bounded support, endowed with a certain degree of differentiability. In this direction, in [15] it is proved that the existence of a bump function which is $n$-times Fréchet differentiable with the last derivative $(p-n)$-Hölder continuous on a Banach space $X$, not containing copies of $\ell_{2 k}$, implies the existence of upper $p$-estimates in the sequences of the space. Again, since most of r.i. function spaces contain $\ell_{2}$ this kind of results do not give very precise information on these spaces. Our approach here is to consider disjoint sequences in the space. In this sense, we prove that if a Banach lattice $X$ does not contain a vector sublattice order-isomorphic to $\ell_{2 k}$ and admits a bump function which is $n$-times Fréchet differentiable with the last derivative $(p-n)$-Hölder continuous, then $X$ has 
$U D S_{p}$-property. Some related results are also obtained. Finally, we investigate the best order of smoothness of Lorentz function spaces $L_{p, q}$.

\section{2. $U D S_{p}, U D T_{p}$-PROPERTIES AND BOYD INDICES.}

We first give some definitions. We refer to [21] for details. Recall that if $X(I)$ is an r.i. function space on an interval $I$ which is either $[0,1]$ or $[0, \infty)$, the Boyd indices $p_{X}, q_{X}$ are defined by

$$
p_{X}=\lim _{s \rightarrow \infty} \frac{\log s}{\log \left\|D_{s}\right\|} \quad q_{X}=\lim _{s \rightarrow 0^{+}} \frac{\log s}{\log \left\|D_{s}\right\|},
$$

where for each $0<s<\infty$, the linear operator $D_{s}: X(I) \rightarrow X(I)$ is defined by:

(1) In the case of $I=[0, \infty)$, for a measurable function $f$ on $I$ :

$$
\left(D_{s} f\right)(t)=f(t / s), \quad 0 \leq t<\infty
$$

(2) In the case of $I=[0,1]$, for a measurable $f$ on $I$ :

$$
\left(D_{s} f\right)(t)=f(t / s) \chi_{[0, \min \{1, s\}]},, \quad 0 \leq t \leq 1 .
$$

The Boyd indices can be also defined for r.i. spaces on the integers. In this case the operators $D_{s}$ are defined only if $s$ is an integer or the reciprocal of an integer. If $f=\left(a_{1}, a_{2}, \ldots,\right)$ and $n=1,2, \ldots$ we consider :

$$
\begin{aligned}
D_{n} f & =\left(a_{1}, \ldots, a_{1}, a_{2}, \ldots, a_{2}, \ldots\right) \\
D_{1 / n} f & =n^{-1}\left(\sum_{i=1}^{n} a_{i}, \sum_{i=n+1}^{2 n} a_{i}, \ldots\right) .
\end{aligned}
$$

The indices $p_{X}$ and $q_{X}$ are defined as above by taking the limits only over $s=n$ (respectively, $s=1 / n), n=1,2, \cdots$.

Now consider $X(\mathbb{N})$ a separable r.i. space on $\mathbb{N}$. Note that in these spaces the basis $\left\{e_{n}\right\}=\left\{\chi_{\{n\}}\right\}$ is a symmetric sequence, and therefore such spaces coincide with spaces having symmetric basis. We have:

Proposition 1. Let $X(\mathbb{N})$ be a separable r.i. space on $\mathbb{N}$ and let $1<p, q<\infty$.

(1) If $X(\mathbb{N})$ has $U D S_{p}$-property, then $p \leq p_{X}$.

(2) If $X(\mathbb{N})$ has $U D T_{q}$-property, then $q \geq q_{X}$. 
ESTIMATES OF DISJOINT SEQUENCES IN BANACH LATTICES AND R.I. FUNCTION SPACES

Proof. We give the proof in the case of upper estimates; the result for lower estimates is obtained in an analogous way.

Consider $e_{n}=\chi_{\{n\}}$ for all $n \in \mathbb{N}$. Since the space has $U D S_{p}$-property, there is a constant $C>0$ such that each normalized disjoint sequence in $X(\mathbb{N})$ admits a subsequence with an upper $p$-estimate with constant $C$. Let $x=a_{1} e_{1}+\cdots+a_{k} e_{k} \in$ $X(\mathbb{N})$ with $\|x\|=1$. Then,

$$
D_{n}(x)=a_{1}\left(e_{1}+\cdots+e_{n}\right)+\cdots+a_{k}\left(e_{(n-1) k+1}+\cdots+e_{n k}\right)=x_{1}+\cdots+x_{n}
$$

where

$$
\begin{gathered}
x_{1}=a_{1} e_{1}+a_{2} e_{n+1}+\cdots+a_{k} e_{n(k-1)+1} \\
x_{n}=a_{1} e_{n}+a_{2} e_{2 n}+\cdots+a_{k} e_{k n}
\end{gathered}
$$

and $\left\|x_{1}\right\|=\cdots=\left\|x_{n}\right\|=1$. Consider now, $x_{n+i}=a_{1} e_{(n+i-1) k+1}+\cdots+$ $a_{k} e_{(n+i-1) k+k}$ for $i=1,2, \ldots$ The sequence $\left\{x_{n}\right\}_{n=1}^{\infty}$ is normalized and disjoint and therefore it admits a subsequence $\left\{x_{n_{k}}\right\}_{k=1}^{\infty}$ with an upper $p$-estimate with constant $C$. Thus,

$$
\left\|x_{k_{1}}+\cdots+x_{k_{n}}\right\| \leq C n^{1 / p}
$$

and consequently,

$$
\left\|x_{1}+\cdots+x_{n}\right\|=\left\|x_{k_{1}}+\cdots+x_{k_{n}}\right\| \leq C n^{1 / p}
$$

Thus, $\left\|D_{n} x\right\| \leq C n^{1 / p}$ where $C$ is independent of $n$. Now, since the space is separable, sequences with finite support are dense in the space, and therefore for every $n \in \mathbb{N}$ we have $\left\|D_{n}\right\| \leq C n^{1 / p}$. It is easy to check now that

$$
p_{X}=\lim _{n \rightarrow \infty} \frac{\log n}{\log \left\|D_{n}\right\|} \geq p
$$

as we required.

Now we would like to recall that, if a Banach lattice $X$ does not contain a vector sublattice order-isomorphic to $\ell_{1}$, then every normalized disjoint sequence in $X$ is weakly null. This is a consequence (taking positive and negative parts) of the following well-known result, which will be useful along the paper. For a proof, see e.g [1], Theorem 14.21. 
Proposition 2. Let $\left\{x_{n}\right\}_{n=1}^{\infty}$ be a normalized disjoint sequence of positive vectors in a Banach lattice $X$. The following are equivalent:

(1) $\left\{x_{n}\right\}_{n=1}^{\infty}$ is not weakly null.

(2) $\left\{x_{n}\right\}_{n=1}^{\infty}$ contains a subsequence equivalent to the unit vector basis of $\ell_{1}$.

Suppose now that $X(\mathbb{N})$ is a separable r.i. space on $\mathbb{N}$, not containing any vector sublattice order-isomorphic to $\ell_{1}$. Then every normalized disjoint sequence is weakly null and, conversely, every weakly null normalized sequence is equivalent to a disjoint sequence. On the other hand, by [19], if the space has $S_{p}$-property then there is a constant $C>0$ such that every weakly null sequence has a subsequence with an upper $p$-estimate with the same constant $C$. Therefore in this case $S_{p}$-property and $U D S_{p}$-property coincide, and $\ell_{d}(X)$ coincides with the index $\ell(X)=\sup \left\{p \geq 1 ; \quad X\right.$ has $S_{p}$ - property $\}$ introduced in [16]. That is, we have in this case $\ell(X)=\ell_{d}(X) \leq p_{X}$. In some cases we have equality. For example, if $X=\ell_{M}$ is a separable Orlicz sequence space with $1<p_{X}<q_{X}<\infty$, it is shown in [14 that $\ell\left(\ell_{M}\right)=\alpha_{X}=p_{X}$ (see also [21] Proposition 2.b.5). In general, $p_{X}$ is strictly larger than $\ell(X)$, as the following example shows.

Recall that for $1<p, q<\infty$ the Lorentz sequence space $X=\ell_{p, q}$ is defined as the space of all sequences $\left\{a_{n}\right\}_{n=1}^{\infty}$ for which

$$
\left\|\left(a_{n}\right)_{n=1}^{\infty}\right\|_{p, q}=\left(\sum_{n=1}^{\infty}\left(a_{n}^{*}\right)^{q}\left(n^{q / p}-(n-1)^{q / p}\right)\right)^{1 / q}<\infty
$$

where $\left\{a_{n}^{*}\right\}_{n=1}^{\infty}$ is the decreasing rearrangement of $\left\{\left|a_{n}\right|\right\}_{n=1}^{\infty}$.

Example 1. For $1<q<p<\infty$, the Lorentz sequence space $X=\ell_{p, q}$ satisfies $\ell(X)=\ell_{d}(X)=q<p=p_{X}$.

Proof. In the case $1<q<p<\infty$ we have that the Lorentz space $\ell_{p, q}$ coincides with the Lorentz sequence space $d(w ; q)$ with weights $w_{n}=n^{q / p}-(n-1)^{q / p}$, and it is reflexive (see [20] pg. 178). In particular, it does not contain any copy of $\ell_{1}$. An easy computation shows that in these spaces $\left\|D_{n}\right\|=n^{1 / p}$ for each integer $n$. Consequently both Boyd indices coincide with $p$, that is, $p_{X}=q_{X}=p$. On the other hand, as shown in [14], $\ell(d(w ; q))=q$. 
Now we turn to the case of separable r.i. function spaces on an infinite interval $X[0, \infty)$.

Proposition 3. Let $X[0, \infty)$ be a separable r.i. function space on $[0, \infty)$, and consider $1<p, q<\infty$.

(1) If $X[0, \infty)$ has $U D S_{p}$-property, then $p \leq p_{X}$.

(2) If $X[0, \infty)$ has $U D T_{q}$-property, then $q \geq q_{X}$.

Proof. We will prove (1), and (2) is analogous. Assume that $X[0, \infty)$ has $U D S_{p^{-}}$ property with constant $C>0$. First of all, since $X[0, \infty)$ is a separable r.i. function space, step functions are dense on $X[0, \infty)$ and therefore, it is enough to show that there is a constant $R>0$ such that

$$
\left\|D_{s}(f)\right\| \leq R s^{1 / p} \quad \text { if } \quad s \geq 1
$$

for every norm-one step function $f$.

Now let $f=\sum_{i=1}^{m} \lambda_{i} \chi_{\left[u_{i-1}, u_{i}\right)}$, where $0=u_{0}<u_{1}<\cdots<u_{m}=r$, with $\|f\|=1$. We consider for all $k \in \mathbb{N}$,

$$
f_{n}=\sum_{i=1}^{m} \lambda_{i} \chi_{\left[(n-1) r+u_{i-1},(n-1) r+u_{i}\right)}
$$

Then, the sequence $\left\{f_{n}\right\}_{n=1}^{\infty}$ constructed in such a way is normalized and disjoint, and therefore it admits a subsequence $\left\{f_{n_{k}}\right\}_{k=1}^{\infty}$ with an upper $p$-estimate. Thus,

$$
\left\|f_{n_{1}}+\cdots+f_{n_{k}}\right\| \leq C k^{1 / p}
$$

and

$$
\left\|f_{1}+\cdots+f_{k}\right\|=\left\|f_{n_{1}}+\cdots+f_{n_{k}}\right\| \leq C k^{1 / p}
$$

Moreover, with a suitable automorphism $\sigma$ of $[0, \infty)$ we have that $f_{1}+\cdots+f_{k}=$ $g \circ \sigma$ where

$$
g=\lambda_{1} \chi_{\left[0, k u_{1}\right)}+\lambda_{2} \chi_{\left[k u_{1}, k u_{2}\right)}+\cdots+\lambda_{m} \chi_{\left[k u_{m-1}, k u_{m}\right)} .
$$

Now $g=D_{k} f$ since

$$
\left(D_{k} f\right)(t)=f(t / k) .
$$


Thus,

$$
\left(D_{k} f\right)(k x)=f(k x / k)=f(x)
$$

and

$$
\|g\|=\left\|D_{k} f\right\|=\left\|f_{1}+f_{2}+\cdots+f_{k}\right\| \leq C k^{1 / p}
$$

for all $k \in \mathbb{N}$. As a consequence,

$$
\left\|D_{k} f\right\| \leq C k^{1 / p}
$$

for all $k \in \mathbb{N}$. Next, if $s>1$, there is an integer $k$ such that $k \leq s<k+1$. Since $D_{s} f \leq D_{k+1} f$ we have that

$$
\begin{gathered}
\left\|D_{s} f\right\| \leq\left\|D_{k+1} f\right\| \leq C(k+1)^{1 / p}\|f\|= \\
C\left(\frac{k+1}{k}\right)^{1 / p} k^{1 / p}\|f\| \leq C 2^{1 / p} s^{1 / p}
\end{gathered}
$$

Therefore, there is a constant $R>0$ such that

$$
\left\|D_{s}(f)\right\| \leq R s^{1 / p}
$$

for any step function $f$ with $\|f\|=1$.

Now, it is easy to check that:

$$
p_{X}=\lim _{s \rightarrow \infty} \frac{\log s}{\log \left\|D_{s}\right\|} \geq p
$$

as we required.

As a consequence, we obtain:

Corollary 1. Let $X[0, \infty)$ be a separable r.i. function space. Then:

(1) $u p(X) \leq \ell_{d}(X) \leq p_{X}$.

(2) $\operatorname{low}(X) \geq u_{d}(X) \geq q_{X}$.

For separable Orlicz function spaces $X=L_{M}[0, \infty)$ we obtain equalities above. Indeed, it is shown in 21] (Proposition 2.b.5) that in this case $p_{X}=u p(X)$ and $q_{X}=\operatorname{low}(X)$.

On the other hand, in the case of separable Orlicz function spaces $L_{M}[0, \infty)$, it is possible to obtain the following characterization of $U D S_{p}$ and $U D T_{q}$ properties in terms of the Orlicz function $M$ : 
ESTIMATES OF DISJOINT SEQUENCES IN BANACH LATTICES AND R.I. FUNCTION SPACE9

Proposition 4. Let $L_{M}[0, \infty)$ be a separable Orlicz function space. The following statements are equivalent:

(1) $L_{M}[0, \infty)$ has $U D S_{p}$-property (respectively, $U D T_{q}$-property).

$$
\inf _{t>0, \lambda \geq 1} \frac{M(\lambda t)}{\lambda^{p} M(t)}>0 \quad\left(\text { resp. } \sup _{t>0, \lambda \geq 1} \frac{M(\lambda t)}{\lambda^{q} M(t)}<\infty\right)
$$

Proof. We prove the result concerning the $U D S_{p^{-}}$property. The proof for $U D T_{q^{-}}$ property is analogous. Proceeding as in the proof of Proposition 3, we have that there is a constant $R>0$ such that $\left\|D_{s}\right\| \leq R s^{1 / p}$. In particular, if $u>0$ for $f=\chi_{[0, u / s]}$ we have:

$$
\left\|\chi_{[0, u]}\right\| \leq R s^{1 / p}\left\|\chi_{[0, u / s]}\right\|
$$

We now proceed now as in 21] (pg. 139). Let $u>0$ and $s \geq 1$ be fixed; since

$$
M^{-1}\left(\frac{1}{u}\right)=\frac{1}{\left\|\chi_{[0, u]}\right\|}
$$

we have:

$$
M^{-1}\left(\frac{s}{u}\right)=\frac{1}{\left\|\chi_{[0, u / s]}\right\|} \leq R s^{1 / p} \frac{1}{\chi_{[0, u]}}=R s^{1 / p} M^{-1}\left(\frac{1}{u}\right) .
$$

By taking: $t=M^{-1}\left(\frac{1}{u}\right)$ and $\lambda=R s^{1 / p}$, it follows that if $t>0$ and $\lambda \geq R$ :

$$
M(\lambda t) \geq s / u=M(t) \lambda^{p} \frac{1}{R^{p}}
$$

and therefore,

$$
\inf _{t>0, \lambda \geq 1} \frac{M(\lambda t)}{M(t) \lambda^{p}}>0
$$

as we required.

In order to prove that (2) implies (1), by using [21] (see pg. 140) we obtain that if:

$$
\inf _{t>0, \lambda \geq 1} \frac{M(\lambda t)}{M(t) \lambda^{p}}>0
$$

then the space $L_{M}[0, \infty)$ has an upper $p$-estimate, and consequently it has $U D S_{p^{-}}$ property. 
Now we give an application, taking into account that, for $1<p, q<\infty$ the function spaces $L_{p}[0, \infty) \cap L_{q}[0, \infty)$ and $L_{p}[0, \infty)+L_{q}[0, \infty)$ can be described as Orlicz spaces. Namely, we have that $L_{p}[0, \infty) \cap L_{q}[0, \infty)=L_{M}[0, \infty)$, where $M(t)=\min \left\{t^{p}, t^{q}\right\}$ and $L_{p}[0, \infty)+L_{q}[0, \infty)=L_{M}[0, \infty)$, where $M(t)=$ $\max \left\{t^{p}, t^{q}\right\}$. Therefore, with a simple calculation we obtain:

Corollary 2. Let $1<p, q<\infty$. Then,

(i) The spaces $L_{p}[0, \infty) \cap L_{q}[0, \infty)$ and $L_{p}[0, \infty)+L_{q}[0, \infty)$ have $U D S_{r^{-}}$ property if and only if $r \leq \min \{p, q\}$.

(ii) The spaces $L_{p}[0, \infty) \cap L_{q}[0, \infty)$ and $L_{p}[0, \infty)+L_{q}[0, \infty)$ have $U D T_{r^{-}}$ property if and only if $r \geq \max \{p, q\}$.

To finish this section, we see that the situation is quite different in the case of r.i. function spaces on the interval $[0,1]$. First recall that, if $1<p, q<\infty$ and $I$ is either $[0,1]$ or $[0, \infty)$, the Lorentz space $L_{p, q}(I)$ is the space of all measurable functions $f$ on $I$ for which

$$
\|f\|_{L_{p, q}}=\left(\int_{I}\left(f^{*}(t)\right)^{q} d\left(t^{\frac{q}{p}}\right)\right)^{\frac{1}{q}}<\infty,
$$

where $f^{*}$ denotes the decreasing rearrangement of $f$. We are grateful to E. Semenov for kindly providing us the following result.

Proposition 5. Let $1<p, q<\infty$, and consider the space $X=L_{p, q}[0,1]$. Then:

(1) $X$ has Boyd indices $p_{X}=q_{X}=p$.

(2) $X$ satisfies that $u p(X)=\min \{p, q\}$ and $\operatorname{low}(X)=\max \{p, q\}$.

(3) $X$ has both $U D S_{q}$ and $U D T_{q}$-properties, and therefore

$$
\ell_{d}(X)=u_{d}(X)=q
$$

Proof. For (1) we refer to [3] and [4]; and (2) can be seen in [8]. Now we pass to (3). For the case $1<q<p<\infty$, it is proved in [12] (see Theorem 5.1) that, for each $\varepsilon>0$, every disjoint normalized sequence in $L_{p, q}[0,1]$ admits a subsequence which is $(1-\varepsilon)^{-1}$-equivalent to the unit vector basis of $\ell_{q}$. Consequently, such space verifies $U D S_{q}$ and $U D T_{q}$ properties. On the other hand, for the case 
ESTIMATES OF DISJOINT SEQUENCES IN BANACH LATTICES AND R.I. FUNCTION SPACE\$ $1<q<p<\infty$ it is proved in [5] (see the proof of Theorem 1), that there is a constant $C>0$, only depending on $p$ and $q$, in such a way that for each $\varepsilon>0$, every normalized disjoint sequence in $L_{p, q}[0,1]$ admits an upper $p$-estimate with constant $C=1-2 \varepsilon$. Consequently, the space has $U D S_{q}$-property. Moreover, the space admits a lattice lower $q$-estimate with constant $q$. Consequently, $L_{p, q}[0,1]$ admits $U D S_{q}$ and $U D T_{q}$ properties.

\section{IMPACT OF HIGH ORDER OF SMOOTHNESS ON UPPER ESTIMATES.}

Let $1<p<\infty$ and let $N$ be the greatest integer strictly less than $p$. According to [22], a real valued function $\phi: X \rightarrow \mathbb{R}$ is said to be $U H^{p}$-smooth if it is $N$-times Fréchet differentiable and there is a constant $C>0$ such that if $x, y \in X$,

$$
\left\|\phi^{(N)}(x)-\phi^{(N)}(y)\right\| \leq C\|x-y\|^{p-N} .
$$

As usual, we say that the space $X$ is $U H^{p}$-smooth if there is a $U H^{p}$-smooth function on $X$ with bounded nonempty support.

In the case of separable r.i. spaces $X(\mathbb{N})$, the smoothness of the space has a great impact on its structure as a Banach lattice. More precisely, we have:

Theorem 1. 17] Let $1<p<\infty$ and let $X$ be a Banach space with a symmetric basis, not isomorphic to $\ell_{2 k}$ for $k \in \mathbb{N}$. If $X$ is $U H^{p}$-smooth then it has a lattice upper p-estimate.

This result does not hold in the general case of Banach lattices, as the example $X=\left(\bigoplus_{n=1}^{\infty} \ell_{4}^{n}\right)_{\ell_{6}}$ shows (see [17], Remark 1.6). In order to obtain a variant of Theorem above for Banach lattices, we study the relationship of high order smoothness of the space with the property $U D S_{p}$ introduced in the previous section. We use analogous techniques as those used in [15. We say that a sequence $\left\{x_{n}\right\}_{n=1}^{\infty}$ is $\mathcal{P}_{N}$-null for some integer $N$, if $\lim _{n \rightarrow \infty} P\left(x_{n}\right)=0$ for every homogeneous polynomial on $X$ of degree at most $N$. 
Theorem 2. Let $1<p<\infty$ and let $X$ be a $U H^{p}$-smooth Banach lattice. Then, there is a constant $C>0$ such that, if $\left\{x_{n}\right\}_{n=1}^{\infty}$ is a weakly null, normalized and disjoint sequence in $X$, either

(1) $\left\{x_{n}\right\}_{n=1}^{\infty}$ has a subsequence equivalent to the basis of $\ell_{2 k}$ with $2 k \leq p$, or

(2) $\left\{x_{n}\right\}_{n=1}^{\infty}$ has a subsequence with an upper p-estimate with constant $C$.

Proof. Let $N$ be the greatest integer strictly less than $p$. Let $\phi$ be a $U H^{p}$-smooth function on $X$ verifying that $\phi(0)=0$ and $\phi(x)=2$ for $\|x\| \geq 1$ (this is always possible composing with a suitable smooth real function). Consider $M>0$ such that:

$$
\left\|\phi^{(N)}(x)-\phi^{(N)}(y)\right\| \leq M\|x-y\|^{p-N}
$$

for $x, y \in X$. Let $\left\{x_{n}\right\}_{n=1}^{\infty}$ be a weakly null normalized disjoint sequence in $X$. Two cases may be given:

(1) The sequence $\left\{x_{n}\right\}_{n=1}^{\infty}$ is not $\mathcal{P}_{N}$-null. Then let $r$ be the minimum of all $k \in \mathbb{N}$ such that $\left\{x_{n}\right\}_{n=1}^{\infty}$ is not $\mathcal{P}_{k}$-null, and note that $1<r \leq N<p$. Since the sequence $\left\{x_{n}\right\}_{n=1}^{\infty}$ is unconditional, by Proposition 1.9 in [15] it admits a subsequence with a lower $r$-estimate. On the other hand, by Proposition 1.1. in [15], there exists a subsequence with an upper $r$ estimate, and consequently it is equivalent to the unit vector basis of $\ell_{r}$. Since $X$ is $U H^{p}$-smooth and contains $\ell_{r}$, then $r$ must be an even integer $r=2 k$ (see e.g. [2]). Therefore, the subsequence is equivalent to the unit vector basis of $\ell_{2 k}$ with $2 k \leq N$.

(2) The sequence $\left\{x_{n}\right\}_{n=1}^{\infty}$ is $\mathcal{P}_{N}$-null. Proceeding as in Proposition 1.1. in [15] we obtain a subsequence $\left\{x_{n_{i}}\right\}_{i=1}^{\infty}$ verifying:

$$
\left|\phi\left(\sum_{i=1}^{k} a_{i} f_{n_{i}}\right)-\phi(0)\right| \leq M\left(\sum_{i=1}^{k}\left|a_{i}\right|^{p}\right)+1
$$

for all $a_{1}, \ldots, a_{k} \in \mathbb{R}$ and $k \in \mathbb{N}$. From this we deduce that $\left\{x_{n}\right\}_{n=1}^{\infty}$ has a subsequence with an upper $p$-estimate with constant $C=M^{1 / p}$.

As a consequence we obtain the following: 
ESTIMATES OF DISJOINT SEQUENCES IN BANACH LATTICES AND R.I. FUNCTION SPACES

Corollary 3. Let $1<p<\infty$ and let $X$ be a Banach lattice not containing any vector sublattice order-isomorphic to $\ell_{2 k}$ for $2 k \leq p$. If $X$ is $U H^{p}$-smooth then it has $U D S_{p}$-property.

Proof. Since $X$ is $U H^{p}$-smooth, it is super-reflexive (see e.g. [10], Theorem V.3.2), and in particular it contains no copy of $\ell_{1}$. Now by Proposition 2, every normalized disjoint sequence in $X$ is weakly null, and Theorem 2 applies.

Note that in the above corollary we cannot avoid the requirement for $X$ of not containing a disjoint sequence equivalent to the usual basis of $\ell_{2 k}$. Indeed, for $X=L_{2 k}(\mu)$, with $k \in \mathbb{N}$, we have that $X$ is $U H^{p}$-smooth for every $p>1$ but $X$ has $U D S_{p}$-property only for $p \leq 2 k$.

Corollary 4. Let $X$ be a Banach lattice. If the index $\ell_{d}(X)$ is not an even integer, then the space is not $U H^{q}$-smooth for $q>\ell_{d}(X)$.

Proof. Assume the contrary. Suppose that $\ell_{d}(X)$ is not an even integer and consider $1<p<\ell_{d}(X)<q<\infty$ in such a way that the interval $[p, q]$ does not contain any even integer, and $X$ is $U H^{q}$-smooth. Let $\left\{x_{n}\right\}_{n=1}^{\infty}$ be a normalized disjoint sequence in $X$. Since $X$ has $U D S_{p}$-property, there exists a subsequence with an upper $p$-estimate. Then, $\left\{x_{n}\right\}_{n=1}^{\infty}$ has no subsequence equivalent to the usual basis of $\ell_{2 k}$ with $2 k \leq p$. As in Corollary 3 , since $X$ is $U H^{q}$-smooth it contains no copy of $\ell_{1}$, and by Proposition 2 we have that $\left\{x_{n}\right\}_{n=1}^{\infty}$ is weakly null. Consequently, by Theorem 2 there is a $C>0$ such that $\left\{x_{n}\right\}_{n=1}^{\infty}$ has a subsequence with an upper $q$-estimate with a constant $C$. Then, the space has $U D S_{q}$-property, which is a contradiction.

In [18] it is proved that for Orlicz spaces $\ell_{M}, L_{M}[0,1]$ and $L_{M}[0, \infty)$, the smoothness properties of the space depend on the Boyd indices. In the case of r.i. function spaces on $[0, \infty)$, by using the results obtained in the previous section, we obtain an estimate of the smoothness in terms of the corresponding Boyd index: 
Corollary 5. Let $1<p<\infty$ and let $X[0, \infty)$ be a separable r.i. function space not containing any vector sublattice order-isomorphic to $\ell_{2 k}$ for $2 k \leq p$. If $X[0, \infty)$ is $U H^{p}$-smooth then $p \leq p_{X}$.

In [21] it is proved that if $X$ is a r.i. function space on $I=[0, \infty)$, then for every $1<p<p_{X}$ and $q_{X}<q<\infty$, we have:

$$
L_{p}(I) \cap L_{q}(I) \subset X(I) \subset L_{p}(I)+L_{q}(I)
$$

with the inclusion maps being continuous. The converse of this result is not true in general. As an application of Theorem 2 we may obtain the following result:

Proposition 6. Let $1<p<\infty$.

(1) Suppose that $X(\mathbb{N}) \subset \ell_{p}$ with continuous inclusion, and $X(\mathbb{N})$ is not isomorphic to $\ell_{2 k}$ for $2 k \leq p$. Then $X(\mathbb{N})$ is not $U H^{r}$-smooth for $r>p$.

(2) Suppose that $X[0, \infty) \subset L_{p}[0, \infty)+L_{q}[0, \infty)$ with continuous inclusion, and $X[0, \infty)$ does not contain any vector sublattice order-isomorphic to $\ell_{2 k}$ for $2 k \leq \max \{p, q\}$. Then $X[0, \infty)$ is not $U H^{r}$-smooth for $r>\max \{p, q\}$.

Proof. (1) Note first that $X(\mathbb{N}) \subset \ell_{p}$ with continuous inclusion, means that the basis $\left\{e_{n}\right\}_{n=1}^{\infty}$ of $X(\mathbb{N})$ has a lower $p$-estimate. Assume that $X(\mathbb{N})$ is $U H^{r}$-smooth with $p<r<[p]+1$. Since the basis $\left\{e_{n}\right\}_{n=1}^{\infty}$ is equivalent to all of its subsequences, by Theorem 2 we obtain that $\left\{e_{n}\right\}_{n=1}^{\infty}$ has an upper $r$-estimate, and this is not possible.

(2) Suppose for example that $p<q$, and suppose that $X[0, \infty)$ is $U H^{r}$-smooth for $r>q$. Consider the sequence $f_{n}=\chi_{[n, n+1)}$ in $X[0, \infty)$. In the space $L_{p}[0, \infty)+$ $L_{q}[0, \infty)$, the sequence $\left\{f_{n}\right\}_{n=1}^{\infty}$ is equivalent to the unit vector basis of $\ell_{q}$. Then there exists some $C>0$ such that

$$
C\left(\sum_{n=1}^{\infty}\left|a_{n}\right|^{q}\right)^{1 / q} \leq\left\|\sum_{n=1}^{\infty} a_{n} f_{n}\right\|_{X} .
$$

This means that $\left\{f_{n}\right\}_{n=1}^{\infty}$ admits a lower $q$-estimate and consequently $\left\{f_{n}\right\}_{n=1}^{\infty}$ does not admit any upper $r$-estimate for $r>q$. This is a contradiction by Theorem 2. 
ESTIMATES OF DISJOINT SEQUENCES IN BANACH LATTICES AND R.I. FUNCTION SPACES

A similar result is the following.

Proposition 7. Let $1<p<q<\infty$ and suppose that the interval $[p, q]$ does not contain any even integer.

(1) If $X(\mathbb{N})$ is a r.i. space and $\ell_{p} \subset X(\mathbb{N}) \subset \ell_{q}$ with continuous inclusions, then $X(\mathbb{N})$ is not $U H^{r}$-smooth for $r>q$.

(2) If $X[0, \infty)$ is a r.i. function space and $L_{p}[0, \infty) \cap L_{q}[0, \infty) \subset X[0, \infty) \subset$ $L_{p}[0, \infty)+L_{q}[0, \infty)$ with continuous inclusions, then $X[0, \infty)$ is not $U H^{r}-$ smooth for $r>q$.

Proof. (1) Suppose that $X(\mathbb{N})$ is $U H^{r}$-smooth for $r>q$. Note that since $\ell_{p} \subset$ $X(\mathbb{N}) \subset \ell_{q}$ with continuous inclusions, we have that the symmetric basis $\left\{e_{n}\right\}_{n=1}^{\infty}$ of $X(\mathbb{N})$, has both an upper $p$-estimate and a lower $q$-estimate. Since $[p, q]$ does not contain any even integer, $\left\{e_{n}\right\}_{n=1}^{\infty}$ has no subsequence equivalent to the usual basis of $\ell_{2 k}$ for any $k \in \mathbb{N}$. Therefore, we obtain a contradiction from Theorem 2 .

(2) Suppose that $X[0, \infty)$ is $U H^{r}$-smooth for $r>q$. Consider the sequence $f_{n}=\chi_{[n, n+1)}$ in $X[0, \infty)$. In the space $L_{p}[0, \infty)+L_{q}[0, \infty)$, the sequence $\left\{f_{n}\right\}_{n=1}^{\infty}$ is equivalent to the unit vector basis of $\ell_{q}$, and in the space $L_{p}[0, \infty) \cap L_{q}[0, \infty)$, the sequence $\left\{f_{n}\right\}_{n=1}^{\infty}$ is equivalent to the unit vector basis of $\ell_{p}$. Therefore there exist $C, D>0$ such that:

$$
C\left(\sum_{n=1}^{\infty}\left|a_{n}\right|^{q}\right)^{1 / q} \leq\left\|\sum_{n=1}^{\infty} a_{n} f_{n}\right\|_{X} \leq D\left(\left.\sum_{n=1}^{\infty} a_{n}\right|^{p}\right)^{1 / p} .
$$

That is, $\left\{f_{n}\right\}_{n=1}^{\infty}$ has both an upper $p$-estimate and a lower $q$ estimate and, as before, we obtain a contradiction with Theorem 2 .

In the sequel we investigate the smoothness properties of the Lorentz function spaces. The result for Lorentz sequence spaces was already obtained in [17].

Proposition 8. Let $1<p \neq q<\infty$.

(1) The Lorentz sequence space $\ell_{p, q}$ and the Lorentz function space $L_{p, q}[0, \infty)$ are not $U H^{r}$-smooth if $r>\min \{p, q\}$.

(2) The Lorentz space $L_{p, q}[0,1]$ is not $U H^{r}$-smooth if $r>q$. 
Proof. Since $\ell_{p, q}$ is isometric to a sublattice of $L_{p, q}[0, \infty)$, by [17] it follows that these spaces are not $U H^{r}$-smooth if $r>\min \{p, q\}$.

For $L_{p, q}[0,1]$, it follows from [6] that every disjoint normalized sequence has a subsequence which is equivalent to the unit vector basis of $\ell_{q}$. In the case that $q$ is not an even integer the result follows by using that $\ell_{q}$ is not $U H^{r}$-smooth if $r>q$ (see e.g. [2]).

Otherwise, let $q$ be an even integer and assume that the space is $U H^{q}$-smooth. We now prove that $L_{p, q}[0,1]$ is saturated with subspaces of cotype $q$, that is, every infinite dimensional subspace of $L_{p, q}[0,1]$ contains an infinite dimensional subspace with cotype $q$. Indeed, let $X$ be an arbitrary infinite dimensional subspace of $L_{p, q}[0,1]$ and choose $\left\{f_{n}\right\}_{n=1}^{\infty}$ a weakly null normalized sequence in $X$, which contains no norm convergent subsequence. This is always possible since $L_{p, q}[0,1]$ is super-reflexive. By using an idea of Rabiger (see [23]) one can easily prove that passing to a subsequence $\left\{f_{n}\right\}_{n=1}^{\infty}$ either has a lower 2-estimate or it is equivalent to a disjoint sequence. In the first case, proceeding as in [18] we obtain that the sequence $\left\{f_{n}\right\}_{n=1}^{\infty}$ (passing to a subsequence if necessary) has also an upper 2-estimate; therefore such a sequence is equivalent to the unit vector basis of $\ell_{2}$ and generates a subspace with cotype $q$. In the second case, if the sequence is disjoint, by [6] it has a subsequence equivalent to the unit vector basis of $\ell_{q}$. Since $\ell_{q}$ has cotype $q$ the result follows also in this case. Now, according to [9] the space $L_{p, q}[0,1]$ has a separating polynomial. This is not possible, since by [13] the only r.i. function spaces $X[0,1]$ which admit a separating polynomial are the spaces $L_{2 k}[0,1]$ up to an equivalent norm.

\section{REFERENCES}

[1] Aliprantis, C. and Burkinshaw, O. Positive Operators. Academic Press, 1985.

[2] Bonic, R. and Frampton, J. Smooth functions on Banach manifolds. J. Math. Mech. 15 (1966), 877-898.

[3] Boyd, D. W. The Hilbert transform on rearrangement invariant spaces. Can. J. Math. 19 (1967), 599-616.

[4] Boyd, D. W. Indices of function spaces and their relationship to interpolation. Can. J. Math. 21 (1969), 1245-1254. 
[5] Carothers, N.L. Rearrangement invariant function subspaces of Lorentz function spaces II. Rocky Mountain J. Math. 17 (1987), 607-616.

[6] Carothers, N.L. and Dilworth, S.T. Geometry of Lorentz spaces via interpolation. Longhorn Notes Univ. of Texas 1985-86, 107-134.

[7] Castillo, J.M.F., García, R. and Gonzalo, R. Banach spaces in which all multilinear forms are weakly sequentially continuous. Studia. Math. 136 (1999), 121-145.

[8] Creekmore, J. Type and cotype in Lorentz $L_{p, q}$-spaces. Indagationes. Math. 43 (1981), $141-152$.

[9] Deville, R. Geometrical implications of the existence of very smooth bump functions in Banach spaces. Israel J. Math. 67 (1989), 1-22.

[10] Deville,R., Godefroy, G. and Zizler, V. Smoothness and renormings in Banach spaces. Longman, 1993.

[11] Dineen, S. Complex analysis on infinite-dimensional spaces. Springer-Verlag, 1999.

[12] Fiegel, T., Johnson,W.B. and Tzafriri, L. On Banach Lattices and Spaces having Local Inconditional Structure, with Applications to Lorentz Function Spaces. J. Approx. Theory 13(1975), 395-412.

[13] González, M., Gonzalo, R. and Jaramillo, J. Symetric Polynomials on rearragementinvariant function spaces. J. London Math. Soc. 59 (1999), 681-697.

[14] Gonzalo, R. Upper and lower estimates in Banach sequence spaces. Comment. Math. Univ. Carolinae 36 (1995), 641-653.

[15] Gonzalo, R. and Jaramillo, J.A. Smoothness and estimates of sequences in Banach spaces. Israel J. Math. 89 (1995) 321-341.

[16] Gonzalo, R. and Jaramillo, J.A. Compact polynomials between Banach spaces Proc. Royal Irish Acad. 95A (1995) 213-226.

[17] Gonzalo, R. and Jaramillo, J.A. High order smoothness in sequence spaces and spreading models. Contemporary Math. 232 (1999), 151-160.

[18] Gonzalo, R. and Maleev, R.P. Smooth functions in Orlicz function spaces. Arch. Math. 69 (1997), 136-145.

[19] Knaust, H. and Odell, E. Weakly null sequences with upper $\ell_{p}$-estimates. Lectures Notes in Math. (Springer-Verlag) no. 1470.

[20] Lindenstrauss, J. and Tzafriri, L. Classical Banach spaces I. Springer-Verlag, 1977.

[21] Lindenstrauss, J. and Tzafriri, L. Classical Banach spaces II. Springer-Verlag, 1979.

[22] Meshkov, V. Z. Smoothness properties in Banach spaces. Studia Math. 63 (1978) 111123. 
[23] Rabiger, F. Lower 2-estimates for sequences in Banach lattices Proc. Amer. Math. Soc. 111 (1991), 81-83.

Departamento de Matemática Aplicada, Facultad de Informática de Madrid, Universidad Politécnica, Campus de Montegancedo, Boadilla del Monte, 28660, E-mail address: rngonzalo@fi.upm.es

Departamento de Análisis Matemático, Facultad de Ciencias Matemáticas, Universidad Complutense de Madrid., 28040 Madrid.

E-mail address: jaramil@mat.ucm.es 